\title{
Modeling Nonlinear Acoustooptic Coupling in Fiber Optics Based on Refractive Index Variation due to Local Bending
}

\author{
Catalina Hurtado Castano, Rodrigo Acuna Herrera, and Pedro I. Torres \\ Facultad de Ciencias, Escuela de Física, Universidad Nacional de Colombia, Medellín, A.A. 3840, Medellín 20036, Colombia \\ Correspondence should be addressed to Rodrigo Acuna Herrera; racuna@unal.edu.co
}

Received 4 April 2016; Revised 22 August 2016; Accepted 23 August 2016

Academic Editor: José Luís Santos

Copyright ( 2016 Catalina Hurtado Castano et al. This is an open access article distributed under the Creative Commons Attribution License, which permits unrestricted use, distribution, and reproduction in any medium, provided the original work is properly cited.

\begin{abstract}
A detailed procedure is presented to compute analytically the acoustooptic coupling coefficient between copropagating core and lowest-order cladding modes in tapered fiber optics. Based on the effect of the local bending, the linear and nonlinear variations in the refractive index are modeled. A set of equations and parameters are presented in order to calculate the influence of acoustooptic effect in nonlinear pulse propagation. We will show that as the tapered fiber diameter decreases more energy can be transferred to the cladding and the nonlinear phenomena can compensate the coupling coefficients effects.
\end{abstract}

\section{Introduction}

The use of light to solve specific problems has led to the development of new knowledge in materials and optical systems which have found applications in technologies such as photonics [1]. The development of fiber optics (FO) has increased the capacity of processing light by offering systems with ultra high speed such as telecommunication networks and fiber laser [2,3]. Furthermore, FO can be used as a multiplexers, modulators, and tunable filters based on acoustooptic (AO) coupling effect.

Although the AO coupling mechanism is well known, the study and design of $\mathrm{AO}$ devices remain as a topic that deserves attention, especially if one wants to achieve high frequency processing and reproducibility. Studies of $\mathrm{AO}$ effect in fibers have been carried out both theoretically and experimentally, where acoustic waves are considered long period fiber grating (LPFG) which are capable of coupling coherent copropagating modes traveling in neighboring wave guide that interact through the evanescent optical field [4]. The phase matching condition between the modes needs the overlapping among the interacting modes, allowing energy transfer between them. LPFGs offer the possibility of nonlinear coupling interaction between copropagating guided modes [5]. LPFGs have been manufactured with UV lasers to study the propagation of nonlinear pulse in coupled modes [6].
Herein, using a local bending model, variations in the refractive index due to the presence of the acoustic wave are calculated. A detailed theory and methodology are presented in order to calculate analytically the nonlinear coupling pulse propagation between copropagating core and linearly polarized (LP) cladding modes in tapered fibers. This paper is structured in the following way: Section 2 presents the fundamental theory of local bending model for linear acoustooptic coupling. Section 3 describes the expressions to calculate field profiles and propagation constants of cladding modes. In Section 4, based on local bending, novel equations for the nonlinear coupling between copropagating core-cladding modes are presented. Section 5 presents the results of numerical simulations. Finally, the conclusions are presented in last section.

\section{Local Bending Model for AO Coupling}

In this section, the $\mathrm{AO}$ coupling interactions between the fundamental core mode and copropagating cladding modes are analyzed. We see that acoustooptic coupling equations described changes in the amplitude of the mode propagated through an optical fiber, either in core region or in cladding region.

The transference of energy between fiber modes can be achieved by the presence of acoustic waves [7]; we assume 
that a single mode fiber is modulated in the $x-z$ plane and the modulation locally bends it with radius of $R(z)$. The effect of the local bending, as a consequence of acoustic wave, can be modeled as a variation in the refractive index [8] of the fiber which is given by the expression

$$
n^{2}=n_{\mathrm{co}}^{2}\left[1-(1+\chi) \frac{2 x}{R(z)}\right] \text {, }
$$

where $n_{\text {co }}$ is the core refractive index for the fundamental fiber mode without bending, $x$ is the local coordinate on the straight fiber, and $\chi$ is the silica elastooptic coefficient whose value is -0.22 . Assuming that the amplitude of oscillation is much smaller than the acoustic wavelength, one can approximate the local curvature $\kappa=1 / R(z)$ as

$$
\kappa \approx A \Omega^{2} \cos (\Omega z)
$$

where $A$ is acoustic amplitude, $\Omega=2 \pi / \Lambda$ is the acoustic wave number, and $\Lambda$ is acoustic wave period. The coupled-mode equations that describe linear coupling interactions in a longperiod grating are

$$
\begin{aligned}
& \frac{\partial a^{\mathrm{co}}}{\partial z}=i C_{\mathrm{co}-\mathrm{cl}} e^{i \beta_{0 \nu} z} a_{\nu}^{\mathrm{cl}}, \\
& \frac{\partial a_{v}^{\mathrm{cl}}}{\partial z}=i C_{\mathrm{cl}-\mathrm{co}} e^{i \beta_{0 \nu} z} a^{\mathrm{co}},
\end{aligned}
$$

where $a^{\text {co }}$ is the core mode amplitude, $a_{v}^{\mathrm{cl}}$ the cladding $\nu$ mode amplitude, and $\beta_{0 v}$ the detuning parameter. In (4) the longitudinal part of the coupling coefficient is neglected since it is substantially much more smaller than the transverse part [9]. The maximum transference of energy is given at the phase condition $\beta_{0 \nu}\left(\lambda_{r}\right)=0$, where $\lambda_{r}$ is the resonant wavelength. Then, we can write

$$
\beta_{0 \nu}\left(\lambda_{r}\right)=\beta^{\mathrm{co}}\left(\lambda_{r}\right)-\beta_{v}^{\mathrm{cl}}\left(\lambda_{r}\right)-\Omega
$$

The simplified expression of the coupling coefficient between the fundamental core mode and any cladding mode is given by $C_{\mu \nu}$ and described in [8] for local bending model; an extensive expression can be seen in [10, Eq. (34)]. In this paper, we consider the interaction between the fundamental core mode $\left(\mathrm{LP}_{01}^{\mathrm{co}}\right)$ and the first three lowest cladding modes $\left(\mathrm{LP}_{1 \nu}^{\mathrm{cl}}, v=1,2,3\right)$ since $C_{\mu \nu}$ is only nonzero for modes that differ by an odd integer in angular momentum [8]. Using the local bending model, $C_{\mu \nu}$ is written as

$$
C_{\mu \nu}=\frac{A k_{o}^{2} n_{\mu}^{2} \Omega^{2}(1+\chi)}{2 \beta_{\mu}}\left\langle\xi_{\mu t}|x| \xi_{v t}\right\rangle,
$$

where subscripts $\mu$ and $v$ reference the core and cladding mode, respectively; $\xi_{\mu t}$ is the transverse mode profile, normalized according to $\left\langle\xi_{\mu t} \mid \xi_{v t}\right\rangle=\delta_{\mu v}$. The normalized power carried by the $\mathrm{LP}_{01}^{\mathrm{co}}$ and $\mathrm{LP}_{1 v}^{\mathrm{cl}}$ modes can be written as a function of the propagation distance $z$ as

$$
\begin{aligned}
& \frac{P^{\mathrm{co}}(z)}{P^{\mathrm{co}}(0)}=\cos ^{2}\left(\gamma_{0 \nu} z\right)+\left(\frac{\beta_{0 \nu}}{2 \gamma_{0 \nu}}\right)^{2} \sin ^{2}\left(\gamma_{0 \nu} z\right), \\
& \frac{P_{\nu}^{\mathrm{cl}}(z)}{P^{\mathrm{co}}(0)}=\left(\frac{\left|C_{0 \nu}\right|}{\gamma_{0 \nu}}\right)^{2} \sin ^{2}\left(\gamma_{0 \nu} z\right),
\end{aligned}
$$

where $\gamma_{0 v}^{2}=\left(\beta_{0 v} / 2\right)^{2}+C^{2}, C^{2}=C_{0 v} C_{\nu 0}$, and $P_{v}^{\mathrm{cl}}=\left|a_{\nu}^{\mathrm{cl}}\right|^{2}$. The initial power is pumped through the fundamental core region $P^{\mathrm{co}}(0)=\left|a^{\mathrm{co}}\right|^{2}$, and the initial power is pumped through the cladding region $P_{v}^{\mathrm{cl}}(0)=0$.

\section{LP Cladding Modes}

This section is devoted to describe the expressions to calculate field profiles and propagation constants of cladding modes using exact analytical equations for a step index fiber optics.

The cladding is considered a multimode wave guide; for this to exist, it is necessary to remove the polymer coating to satisfy the condition of total internal reflection at the external boundary [11]; therefore these modes are very sensitive to change because of the surrounding medium. Cladding modes can be excited by using LPFGs which can couple light from core to cladding modes [11]. This happens due to the fact that the condition of total internal reflection is violated when the optical fiber is bending.

We are interested mainly in the interaction between the fundamental core mode $\left(\mathrm{LP}_{01}\right)$ and the lowest $\left(\mathrm{LP}_{1 v}\right)$ cladding modes in a tapered fiber optics. In the case of the mode guided by the fiber core, field profile and propagation constant are calculated using the expressions given in [12]. For cladding modes, we used the dispersion expression given in $[10,13]$ for the three-layer fiber model in order to compute the constant propagations. The cladding mode dispersion equation is given by the equations

$$
\zeta_{0}=\zeta_{0}^{\prime}
$$

where

$$
\begin{aligned}
& \zeta_{0}=\frac{1}{\sigma_{2}} \frac{u_{2}\left(J K+\sigma_{1} \sigma_{2} u_{21} u_{32} / n_{2}^{2} a_{1} a_{2}\right) p_{l}\left(a_{2}\right)-\left(1 / u_{2}\right) s_{l}\left(a_{2}\right)-K q_{l}\left(a_{2}\right)+J r_{l}\left(a_{2}\right)}{\left.\left.-u_{32} / n_{2}^{2} a_{2}\right) J-\left(u_{21} / n_{1}^{2} a_{1}\right) K\right) p_{l}\left(a_{2}\right)-\left(u_{32} / n_{1}^{2} a_{2}\right) q_{l}\left(a_{2}\right)+\left(u_{21} / n_{1}^{2} a_{1}\right) r_{l}\left(a_{2}\right)} \\
& \zeta_{0}^{\prime}=\sigma_{1} \frac{u_{2}\left(\left(u_{32} / a_{2}\right) J-\left(n_{3}^{2} u_{21} / n_{2}^{2} a_{1}\right) K\right) p_{l}\left(a_{2}\right)-\left(u_{32} / a_{2}\right) q_{l}\left(a_{2}\right)-\left(u_{21} / a_{1}\right) r_{l}\left(a_{2}\right)}{u_{2}\left(\left(n_{3}^{2} / n_{2}^{2}\right) J K+\sigma_{1} \sigma_{2} u_{21} u_{32} / n_{1}^{2} a_{1} a_{2}\right) p_{l}\left(a_{2}\right)-\left(n_{3}^{2} / n_{1}^{2}\right) K q_{l}\left(a_{2}\right)+J r_{l}\left(a_{2}\right)-\left(n_{2}^{2} / n_{1}^{2} u_{2}\right) s_{l}\left(a_{2}\right)} .
\end{aligned}
$$



ing:

The variables and functions given in (10) are the follow-

$$
\begin{aligned}
\sigma_{1} & =\frac{i l n_{\mathrm{eff}}}{Z_{0}}, \\
\sigma_{2} & =i \ln _{\mathrm{eff}} Z_{0}, \\
u_{21} & =\frac{1}{u_{2}^{2}}-\frac{1}{u_{1}^{2}}, \\
u_{32} & =\frac{1}{w_{3}^{2}}-\frac{1}{u_{2}^{2}}, \\
u_{j}^{2} & =\left(\frac{2 \pi}{\lambda}\right)^{2}\left(n_{j}^{2}-n_{\mathrm{eff}}^{2}\right) \quad[j \in(1,2)], \\
w_{3}^{2} & =\left(\frac{2 \pi}{\lambda}\right)^{2}\left(n_{\mathrm{eff}}^{2}-n_{3}^{2}\right), \\
J & =\frac{J_{l}^{\prime}\left(u_{1} a_{1}\right)}{u_{1} J_{l}\left(u_{1} a_{1}\right)}, \\
K & =\frac{K_{l}^{\prime}\left(w_{3} a_{2}\right)}{w_{3} K_{l}\left(w_{3} a_{2}\right)}, \\
p_{l}(r) & =J_{l}\left(u_{2} r\right) N_{l}\left(u_{2} a_{1}\right)-J_{l}\left(u_{2} a_{1}\right) N_{l}\left(u_{2} r\right), \\
q_{l}(r) & =J_{l}\left(u_{2} r\right) N_{l}^{\prime}\left(u_{2} a_{1}\right)-J_{l}^{\prime}\left(u_{2} a_{1}\right) N_{l}\left(u_{2} r\right), \\
r_{l}(r) & =J_{l}^{\prime}\left(u_{2} r\right) N_{l}\left(u_{2} a_{1}\right)-J_{l}\left(u_{2} a_{1}\right) N_{l}^{\prime}\left(u_{2} r\right), \\
s_{l}(r) & =J_{l}^{\prime}\left(u_{2} r\right) N_{l}^{\prime}\left(u_{2} a_{1}\right)-J_{l}^{\prime}\left(u_{2} a_{1}\right) N_{l}^{\prime}\left(u_{2} r\right),
\end{aligned}
$$

where $a_{1}$ and $a_{2}$ are the core and cladding radius, respectively. $l$ is the azimuthal number. $J_{l}(x)$ denoted the Bessel functions of the first kind, $N_{l}(x)$ are the Bessel functions of the second kind, and $K_{l}(x)$ are the modified Bessel functions of the second kind; also, $J_{l}^{\prime}(x), N_{l}^{\prime}(x)$, and $K_{l}^{\prime}(x)$ indicate differentiation of Bessel functions with respect to the total argument $x$. Based on the three-layer model, the electric field components for cladding modes are $[10,14]$

$$
\begin{aligned}
(\rho & \left.\leq a_{1}\right) \\
E_{\rho}^{\mathrm{cl}} & =E_{l v}^{\mathrm{cl}} \frac{u_{1}}{2}\left\{J_{l+1}\left(u_{1} \rho\right)+J_{l-1}\left(u_{1} \rho\right)\right. \\
& \left.-\frac{\sigma_{2} \zeta_{0}}{n_{1}^{2}}\left[J_{l+1}\left(u_{1} \rho\right)-J_{l-1}\left(u_{1} \rho\right)\right]\right\} \cos (l \phi), \\
E_{\theta}^{\mathrm{cl}} & =E_{l v}^{\mathrm{cl}} \frac{u_{1}}{2}\left\{J_{l+1}\left(u_{1} \rho\right)-J_{l-1}\left(u_{1} \rho\right)\right. \\
& \left.-\frac{\sigma_{2} \zeta_{0}}{n_{1}^{2}}\left[J_{l+1}\left(u_{1} \rho\right)+J_{l-1}\left(u_{1} \rho\right)\right]\right\} \sin (l \phi), \\
E_{z}^{\mathrm{cl}} & =i E_{l v}^{\mathrm{c}} \frac{\pi a_{1} u_{1}^{2} u_{2}^{2} \sigma_{2} J_{l}\left(u_{1} \rho\right)}{2 n_{2}^{2} \beta l}\left[G_{2} p_{l}(\rho)\right. \\
& \left.-\frac{n_{2}^{2} \xi_{0}}{n_{1}^{2} u_{2}} q_{l}(\rho)\right] \cos (\nu \phi),
\end{aligned}
$$

$$
\begin{aligned}
& F_{2}=J-\frac{u_{21} \sigma_{2} \zeta_{0}}{n_{1}^{2} a_{1}}, \\
& G_{2}=\zeta_{0} J+\frac{u_{21} \sigma}{a_{1}} .
\end{aligned}
$$

The $H E_{2, v}^{\mathrm{cl}}$ conventional mode is equivalent to linearly polarized mode $\mathrm{LP}_{1 v}^{\mathrm{cl}}[12]$, which is our mode of interest. The cladding modes occur in a strict alternating sequence of $H E$ and $E H$, except from the fundamental mode $H E_{1,1}^{\mathrm{cl}}$ [13]. In our case, we set $l=2$ and $v=1,3,5$ in conventional modes which are the first three lowest cladding modes which are the ones we are interested in. Then, Figure 1 shows the $E_{x}$ profile for these modes in a standard tapered single mode fiber optics.

\section{Nonlinear Pulse Propagation in Coupled Copropagating Modes}

Nonlinear pulse propagation in a linear periodic medium has been studied using coupled-mode theory for the copropagating core and cladding modes in a LPFG $[6,15]$, where it is necessary to include both the nonlinear effect and the periodic variation of the refractive index by using the equation

$$
n(\omega, z)=n_{o}+\delta n_{g}(z)+\eta_{2}|E|^{2}=n_{L}+n_{N L},
$$

where $n_{o}$ is the nonperturbed linear refractive index, $\eta_{2}$ is the silica nonlinear refractive index, and $\delta n_{g}(z)$ stands for periodic index variations as a consequence of the grating. If we rewrite the linear part of the refractive index $n_{L}^{2}(\omega, z)=$ $1+f(x, y, z)$, where $f(x, y, z)$ accounts for the refractive index variation associated with the long period grating, one can show that

$$
\begin{aligned}
f(x, y, z) \simeq & \left(n_{o}^{2}-1\right) \\
& \times\left[1+2 n_{o}(1+\chi) A x \Omega^{2} \cos (\Omega z)\right] .
\end{aligned}
$$

Taking (14), using Maxwell's equations, and writing the electric field as a sum of the fundamental core and a LP cladding mode in a fiber optic, one can obtain the nonlinear amplitudes evolution $[6,16]$ :

$$
\begin{aligned}
& \frac{\partial A_{1}}{\partial z}-i \sum_{n=2}^{\infty} \frac{i^{n} \beta_{1 n}}{n !} \frac{\partial^{n} A_{1}}{\partial T^{n}}-i C_{12} A_{2}-i \frac{\delta}{2} A_{1} \\
& \quad=i\left(d_{11}\left|A_{1}\right|^{2}+2 d_{12}\left|A_{2}\right|^{2}\right) A_{1}, \\
& \frac{\partial A_{2}}{\partial z}+V_{g} \frac{\partial A_{2}}{\partial T}-i \sum_{n=2}^{\infty} \frac{i^{n} \beta_{2 n}}{n !} \frac{\partial^{n} A_{2}}{\partial T^{n}}-i C_{12} A_{1}+i \frac{\delta}{2} A_{2} \\
& \quad=i\left(d_{22}\left|A_{2}\right|^{2}+2 d_{12}\left|A_{1}\right|^{2}\right) A_{2} ;
\end{aligned}
$$

here, $A_{1}$ is the amplitude of fundamental core mode and $A_{2}$ the amplitude of a $\mathrm{LP}$ cladding mode $\left(\mathrm{LP}_{11}^{\mathrm{cl}}, \mathrm{LP}_{12}^{\mathrm{cl}}\right.$, or $\left.\mathrm{LP}_{13}^{\mathrm{cl}}\right)$. 


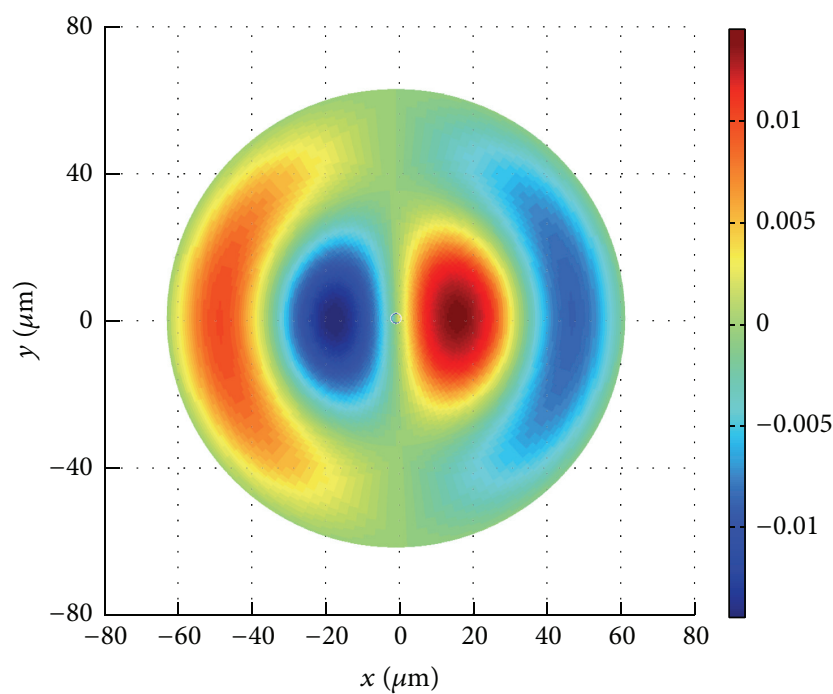

(a) Mode $\mathrm{LP}_{11}^{\mathrm{cl}}$

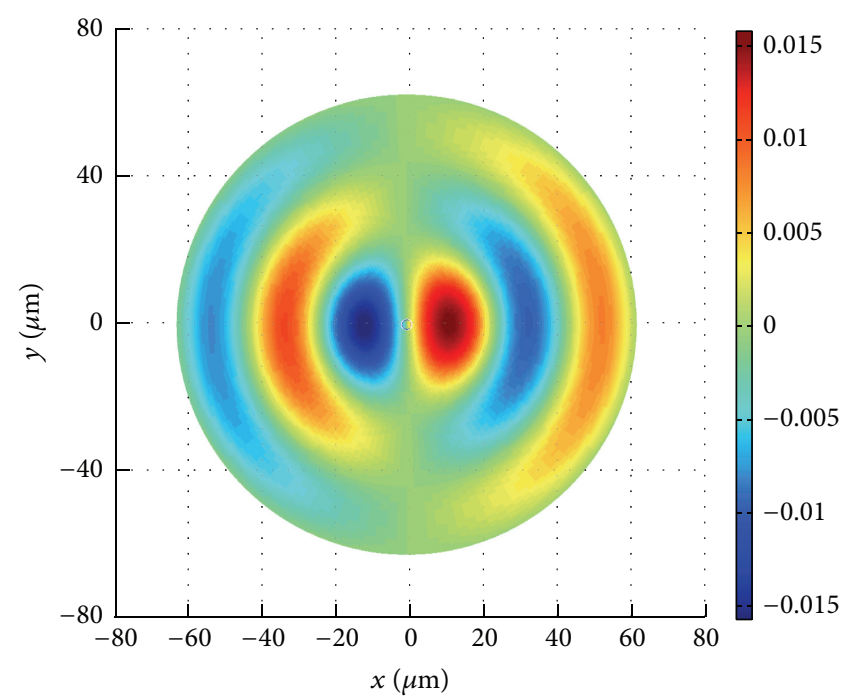

(b) Mode $\mathrm{LP}_{12}^{\mathrm{cl}}$

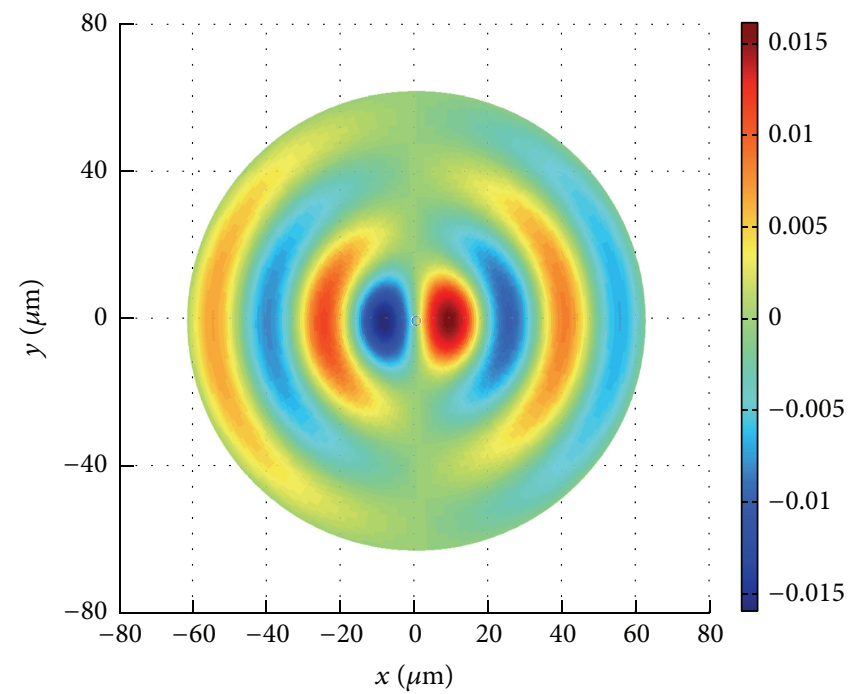

(c) Mode $\mathrm{LP}_{13}^{\mathrm{cl}}$

FIGURE 1: $E_{x}$-profiles for the first three lowest cladding modes $\mathrm{LP}_{11}^{\mathrm{cl}}, \mathrm{LP}_{12}^{\mathrm{cl}}$, and $\mathrm{LP}_{13}^{\mathrm{cl}}$ in a standard tapered single mode fiber optics.

The equation $\beta_{m n}=\partial^{n} \beta_{m} /\left.\partial T^{n}\right|_{\omega_{o}}$ is $n$-order of the Taylor expansion series of $\beta_{m}(m=1,2)$, where $\omega_{o}$ is the pump frequency; $V_{g}=\beta_{21}-\beta_{11}$ and $T=t-\beta_{11} z$. $C_{12}$ is the coupling coefficient given in (6) and $\delta(\lambda)=\beta_{0}^{\mathrm{co}}(\lambda)-\beta_{\nu}^{\mathrm{cl}}(\lambda)-\Omega$ is the detuning, which is the same equation given in (5). The acoustic wave number $(\Omega)$ and acoustic wave period $(\Lambda)$ was described in Section 2. $d_{i j}$ s are the overlapped field coefficients associated with the nonlinear phenomena, given by

$$
d_{i j}=\frac{2 \pi \eta_{2}}{\lambda A_{\mathrm{eff}}} \frac{\left\langle\left(\left|E_{t, i}\right|^{2}\right) \mid\left(\left|E_{t, j}\right|^{2}\right)\right\rangle}{\left\langle\left(\left|E_{t, 1}\right|^{2}\right) \mid\left(\left|E_{t, 1}\right|^{2}\right)\right\rangle}
$$

$E_{t, i}$ is the normalized transverse electric field for the $i$ mode and $A_{\text {eff }}$ is effective fundamental core mode area defined as [16]

$$
A_{\text {eff }}=\frac{\left(\left\langle E_{t, 1} \mid E_{t, 1}\right\rangle\right)^{2}}{\left\langle\left(\left|E_{t, 1}\right|^{2}\right) \mid\left(\left|E_{t, 1}\right|^{2}\right)\right\rangle} .
$$

The coefficients $d_{11}$ and $d_{22}$ are related to self-phase modulation (SPM) for $\mathrm{LP}_{01}^{\mathrm{co}}$ and $\mathrm{LP}_{1 v}^{\mathrm{cl}}$, respectively. $d_{12}$ accounts for cross-phase modulation (XPM) [16].

\section{Numerical Simulations}

The interactions considered in this paper occur between the fundamental core mode $\mathrm{LP}_{01}^{\mathrm{co}}$ and the first three lowest cladding modes $\mathrm{LP}_{1 \mathrm{~m}}^{\mathrm{cl}}$, with $m=1,2,3$. A tapered standard single mode fiber is used for the calculations. Two tapered core diameters are taken into account; they are $d_{\text {co }}=2.5 \mu \mathrm{m}$ and $d_{\text {co }}=3.5 \mu \mathrm{m}$. Fiber reference SMF ITU-T. G.652.B was used. The cladding diameter is reduced in the same 


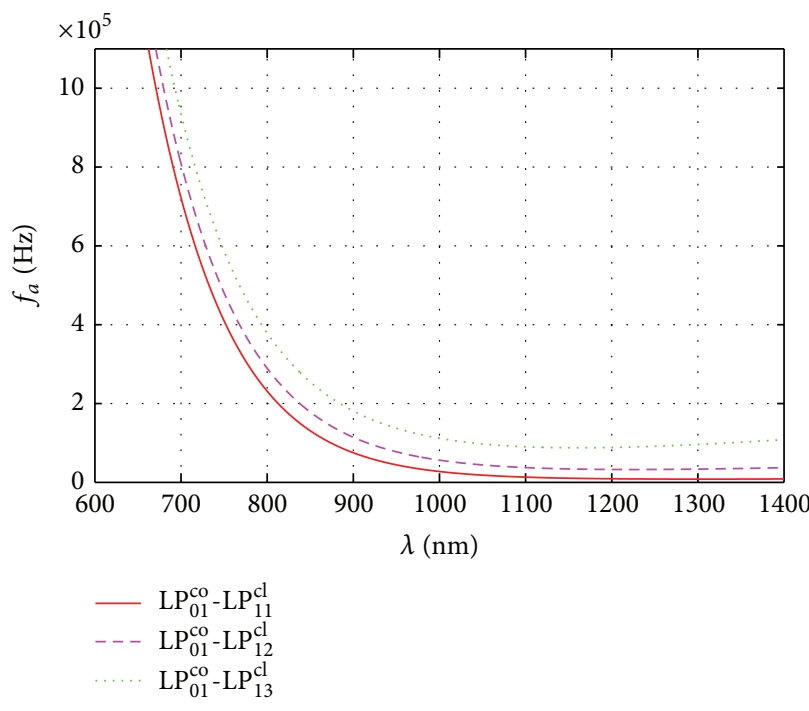

(a) $d_{\text {co }}=2.5 \mu \mathrm{m}$

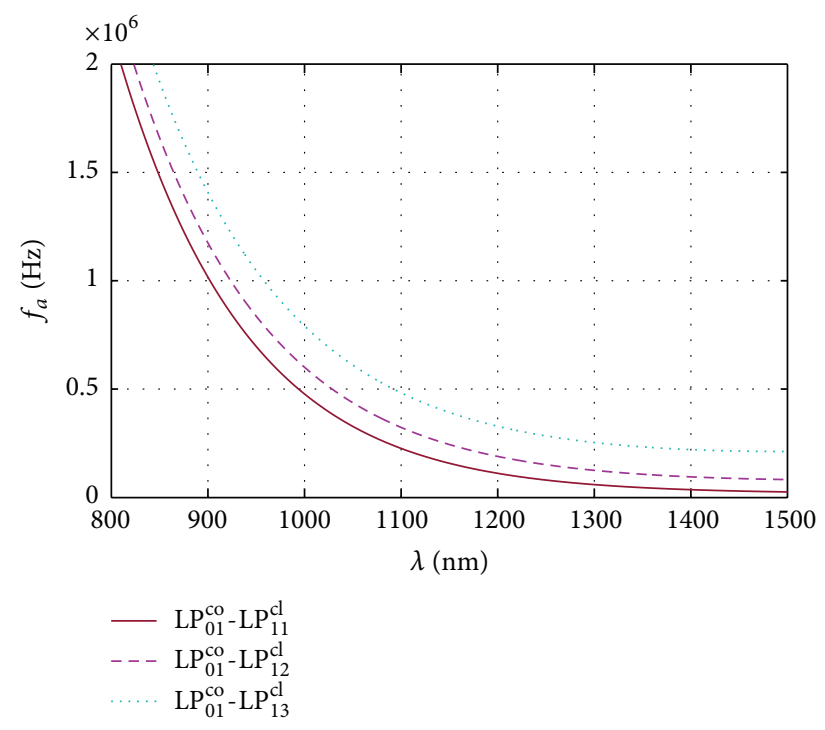

(b) $d_{\mathrm{co}}=3.5 \mu \mathrm{m}$

FIGURE 2: AO frequency $f_{a}$ as a function of wavelength at $\delta(\lambda)=\beta_{0 v}(\lambda)=0$ and coupling between the fundamental core mode and the LP ${ }_{1 \nu}^{\mathrm{cl}} \mathrm{s}$ modes for (a) $d_{\text {co }}=2.5 \mu \mathrm{m}$ and (b) $d_{\text {co }}=3.5 \mu \mathrm{m}$.

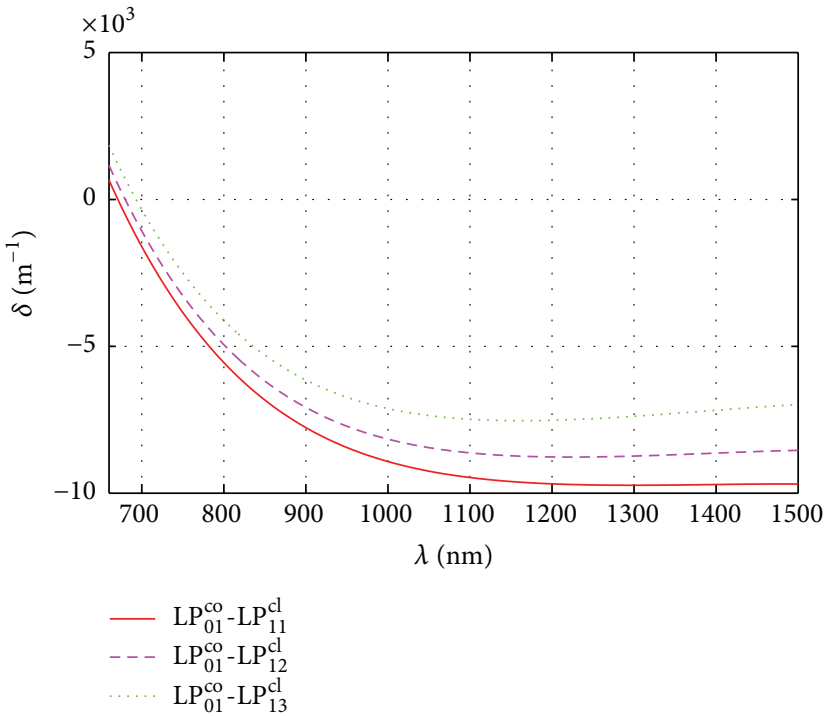

(a) $d_{\mathrm{co}}=2.5 \mu \mathrm{m}$

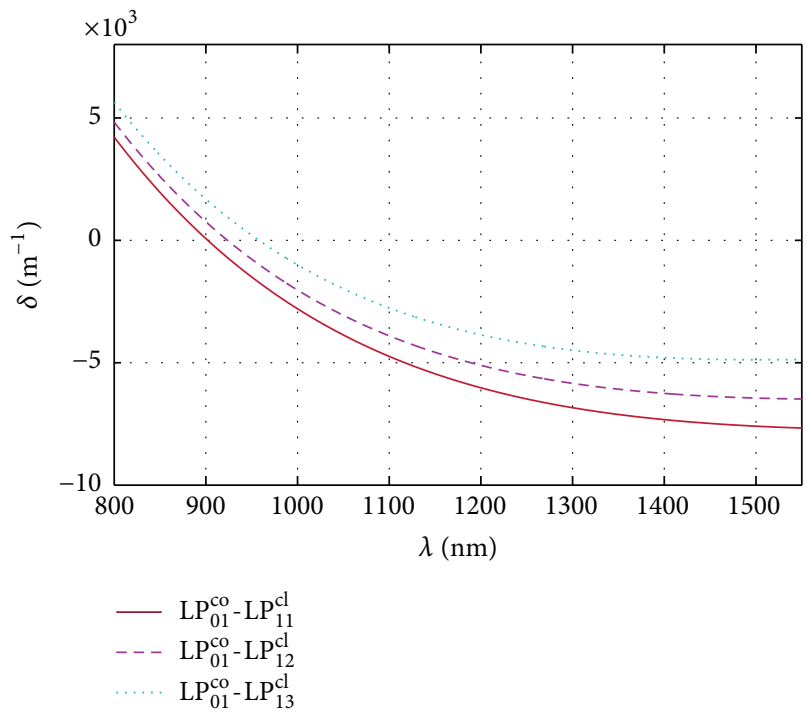

(b) $d_{\mathrm{co}}=3.5 \mu \mathrm{m}$

FIGURE 3: Detuning parameter $\delta$ as a function of wavelength for coupling between the fundamental core mode and the LP $1 \nu^{\mathrm{cl}} \mathrm{s}$ modes when (a) $d_{\text {co }}=2.5 \mu \mathrm{m}$ and (b) $d_{\text {co }}=3.5 \mu \mathrm{m}$.

proportion as the core diameters. Additionally, we set $\Delta=$ $\left(n_{\mathrm{co}}-n_{\mathrm{cl}}\right) / n_{\mathrm{co}}=0.0036$, where $n_{\mathrm{co}}$ stand for the core refractive index and $n_{\mathrm{cl}}$ for the cladding. Sellmeier equation for silica is included.

5.1. Linear Simulations. To compute $C_{12}$ (Eq. (6)) it is necessary to know $\Lambda$ that can be established by the dispersion relationship $[8,17]$ :

$$
f_{a}=\frac{\pi C_{\mathrm{ext}}\left(d_{\mathrm{cl}} / 2\right)}{\Lambda^{2}}
$$

where $f_{a}$ is the acoustic wave frequency, $C_{\text {ext }}=5760 \mathrm{~m} / \mathrm{s}$ is the speed of the acoustic wave in silica, and $d_{\mathrm{cl}}$ is the cladding diameter. It is assumed that axial strain is not applied on the fiber. Figure 2 shows $f_{a}$ as a function of wavelength at $\delta\left(\lambda_{r}\right)=\beta_{0 v}\left(\lambda_{r}\right)=0$ described in (5) and coupling between the fundamental core mode and the $\mathrm{LP}_{1 v}^{\mathrm{cl}} \mathrm{s}$ modes for two tapered fiber diameters. Figure 2 allows us to identify which frequency needs the fiber to oscillate to get a perfect coupling among the modes for a specific wavelength $(\lambda)$. We see that no high $\mathrm{AO}$ frequency is required to transfer efficiently energy from the core to cladding modes when $\lambda$ shifts to infrared. 


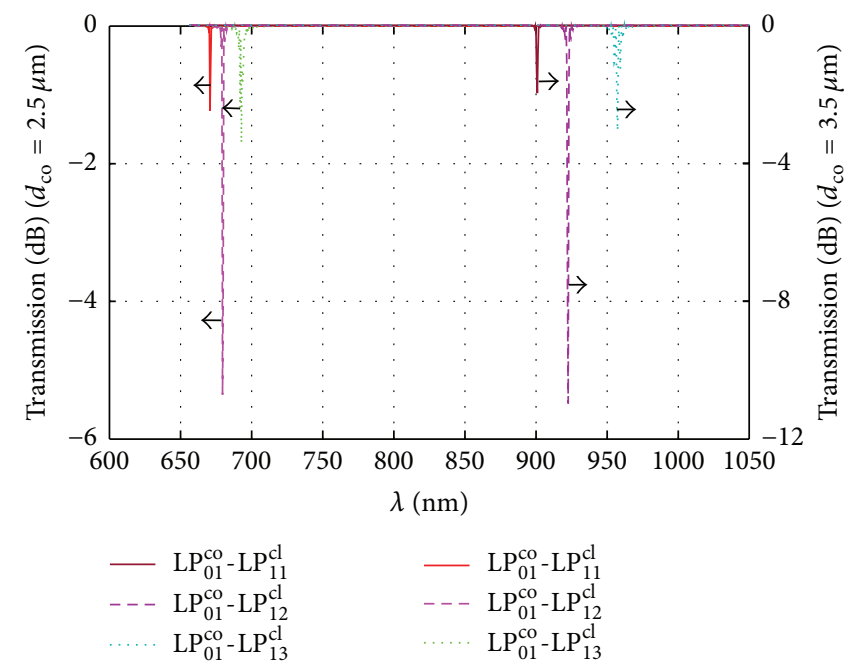

FIGURE 4: Normalized transmitted power in the core mode at the end of the fiber. Setting: fiber length of $L=15 \mathrm{~cm}$, AO wave amplitude of $A=63 \mathrm{~nm}, f_{a}=1 \mathrm{MHz}, d_{\mathrm{co}}=2.5 \mu \mathrm{m}$, and $d_{\mathrm{co}}=3.5 \mu \mathrm{m}$.

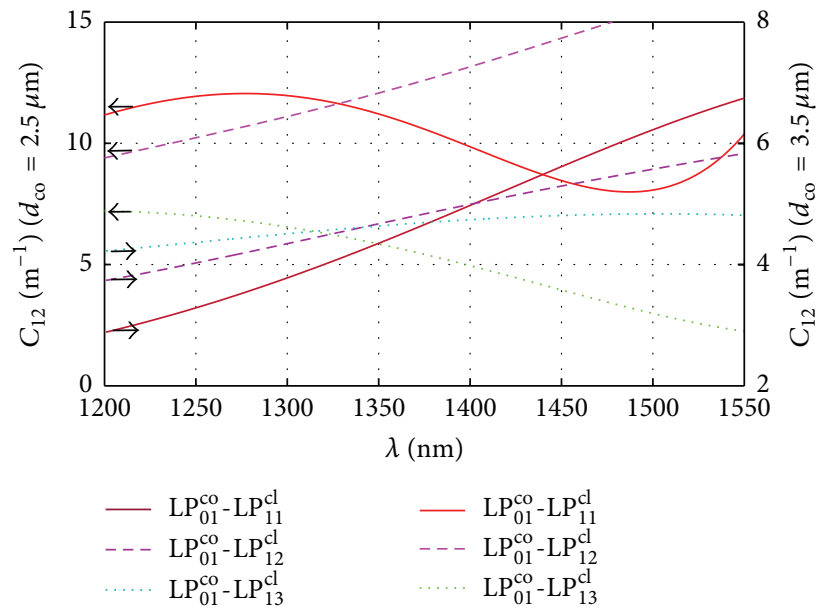

FIGURE 5: AO coupling coefficient $C_{12}$ as a function of wavelength for the coupling between $\mathrm{LP}_{01}^{\mathrm{co}}$ and $\mathrm{LP}_{11}^{\mathrm{cl}}, \mathrm{LP}_{12}^{\mathrm{cl}}$, and $\mathrm{LP}_{13}^{\mathrm{cl}}$ in a tapered standard fiber. Two core diameters are considered, $d_{\text {co }}=2.5 \mu \mathrm{m}$ and $d_{\text {co }}=3.5 \mu \mathrm{m}$.

Additionally, the smaller the tapered fiber diameter is, the more the required $\mathrm{AO}$ frequency decreases, which is a good feature because it could be difficult to find AO drivers at high frequencies.

Fixing AO frequency at $f_{a}=1.0 \mathrm{MHz}$, we compute the detuning $\delta(\lambda)(5)$. The results can be seen in Figure 3. As the tapered diameter decreases the detuning $\delta$ is blue-shifted. $\delta$-zero crossing moves toward blue side around $250 \mathrm{~nm}$ per $1 \mu \mathrm{m}$. At fixed wavelength, $\delta$ increases for higher cladding order modes. From Figures 2 and 3, one can conclude that, by changing the tapered fiber diameter, AO frequency and detuning can be easily controlled, which makes this type of fiber a good candidate to design $\mathrm{AO}$ fiber filter in a large range of wavelengths.

Using (5) and (6), the resulting normalized transmitted power equation (7) in the core mode at the end of the fiber is shown in Figure 4 [18-20], where $P^{\mathrm{co}}(L) / P^{\mathrm{co}}(0)$ in $\mathrm{dB}$ units is plotted. It is set: fiber length of $L=15 \mathrm{~cm}$, AO wave amplitude of $A=63 \mathrm{~nm}$, and $f_{a}=1 \mathrm{MHz}$. According to
(18), $\Lambda=587.20 \mu \mathrm{m}$ for $d_{\text {co }}=2.5 \mu \mathrm{m}$ and $\Lambda=694.79 \mu \mathrm{m}$ for $d_{\text {co }}=3.5 \mu \mathrm{m}$. The coupling between $\mathrm{LP}_{01}^{\mathrm{co}}$ and $\mathrm{LP}_{12}^{\mathrm{cl}}$ exhibits the biggest deep which means that the incident light in the core is partially transferred to the cladding mode at $\lambda=680 \mathrm{~nm}$ for $d_{\text {co }}=2.5 \mu \mathrm{m}$. Similar behavior happens for $d_{\mathrm{co}}=3.5 \mu \mathrm{m}$ at $\lambda=923 \mathrm{~nm}$.

5.2. Nonlinear Simulations. For nonlinear pulse simulations, (15) are numerically solved by using the split-step Fourier method [16]. Fiber length of $15 \mathrm{~cm}$ and initial pulse width of $T_{o}=50 \mathrm{fs}$ are considered. We analyze two scenarios: (i) $d_{\text {co }}=2.5 \mu \mathrm{m}$ : pump wavelength $\lambda_{o}=1323 \mathrm{~nm}$, initial peak power $P_{o}=30 \mathrm{~kW}$, and (ii) $d_{\text {co }}=3.5 \mu \mathrm{m}$ : pump wavelength $\lambda_{o}=1472 \mathrm{~nm}$, initial peak power $P_{o}=3 \mathrm{~kW}$. We choose the above pump wavelengths because we want to generate solitons at both diameters which allow us to get more insight into nonlinear effects.

The linear coupling parameter $C_{12}(\lambda)$ is shown in graph in Figure 5 for the two diameters under consideration. $C_{12}$ 


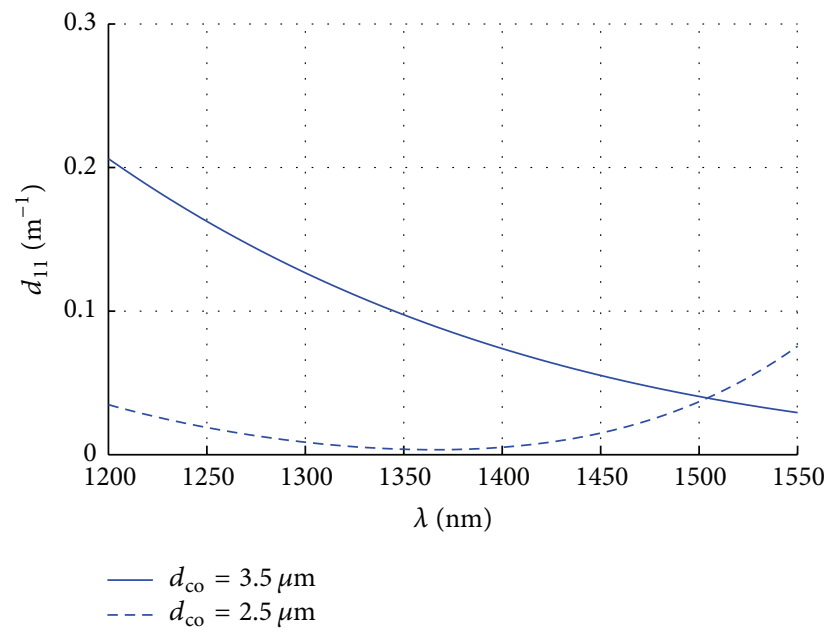

FIGURE 6: Overlap field nonlinear coefficient $d_{11}$ as a function of wavelength in a tapered standard fiber. Two core diameters are considered, $d_{\mathrm{co}}=2.5 \mu \mathrm{m}$ and $d_{\mathrm{co}}=3.5 \mu \mathrm{m}$.

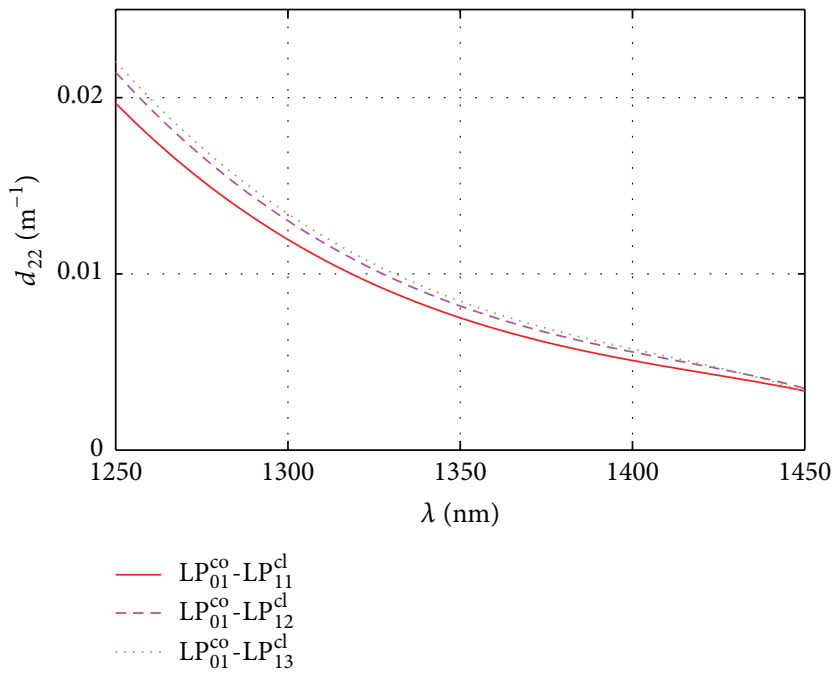

(a) $d_{\mathrm{co}}=2.5 \mu \mathrm{m}$

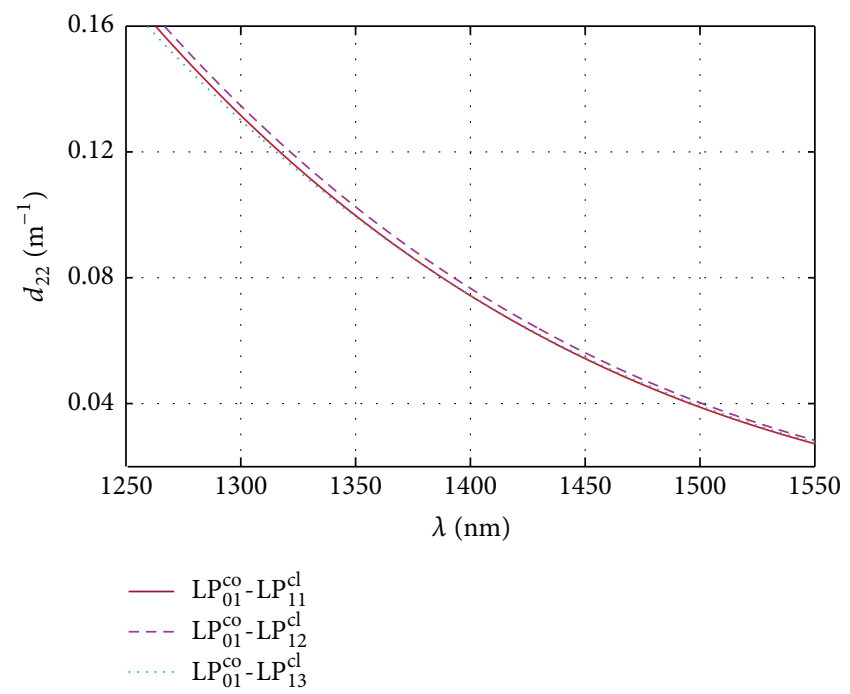

(b) $d_{\text {co }}=3.5 \mu \mathrm{m}$

Figure 7: Overlap field nonlinear coefficient $d_{22}$ as a function of wavelength in a tapered standard fiber. Two core diameters are considered, (a) $d_{\mathrm{co}}=2.5 \mu \mathrm{m}$ and (b) $d_{\mathrm{co}}=3.5 \mu \mathrm{m}$.

almost scales linearly with wavelength, except for $\mathrm{LP}_{01}^{\mathrm{co}}-\mathrm{LP}_{11}^{\mathrm{cl}}$ at $d_{\mathrm{co}}=2.5 \mu \mathrm{m}$ which presents some oscillation. The coupling between $\mathrm{LP}_{01}^{\mathrm{co}}$ and $\mathrm{LP}_{13}^{\mathrm{cl}}$ exhibits the weakest strength at both cores diameters since the mode profiles do not overlap too much.

The plots in Figures 6, 7, and 8 present the overlap nonlinear field coefficients as a function of wavelength between the fundamental core mode and the LP's cladding modes. In Figure 6, we can see that this parameter that governs the SPM phenomenon in the core region is superior for tapered fiber with greater cross-sectional area for most of the analyzed range of wavelength. This could be due to the fact that more energy goes to the cladding as the core diameter is reduced. From Figure $7, d_{22}$ almost linearly decreases as wavelength increases and has the same value for any LP's cladding mode in the same diameter. $d_{22}$ has small value since most of the light keeps in the single mode core, which makes SPM not efficiently excited at low power.

The behavior of the overlap coefficients $d_{12}$ is shown in Figure 8; they also decrease for higher wavelengths. The coefficient $\mathrm{LP}_{01}^{\mathrm{co}}-\mathrm{LP}_{13}^{\mathrm{cl}}$ is the strongest one, which means that more energy can be nonlinearly transferred from the core to the cladding weather; $\mathrm{LP}_{13}^{\mathrm{cl}}$ is efficiently coupled to $\mathrm{LP}_{01}^{\mathrm{co}}$ through the acoustic wave.

To complete the needed parameters to simulate (15), the dispersion coefficients $\beta$ 's are calculated. These can be seen in Table 1 .

Using (15), Figures 9 and 10 display the spectral response of all modes under consideration for both tapered fiber diameters. They evidence the generation of Supercontinuum (SC) both at the core and at the cladding. One can see that SC broadens more at the cladding modes when $d_{\text {co }}=3.5 \mu \mathrm{m}$, 

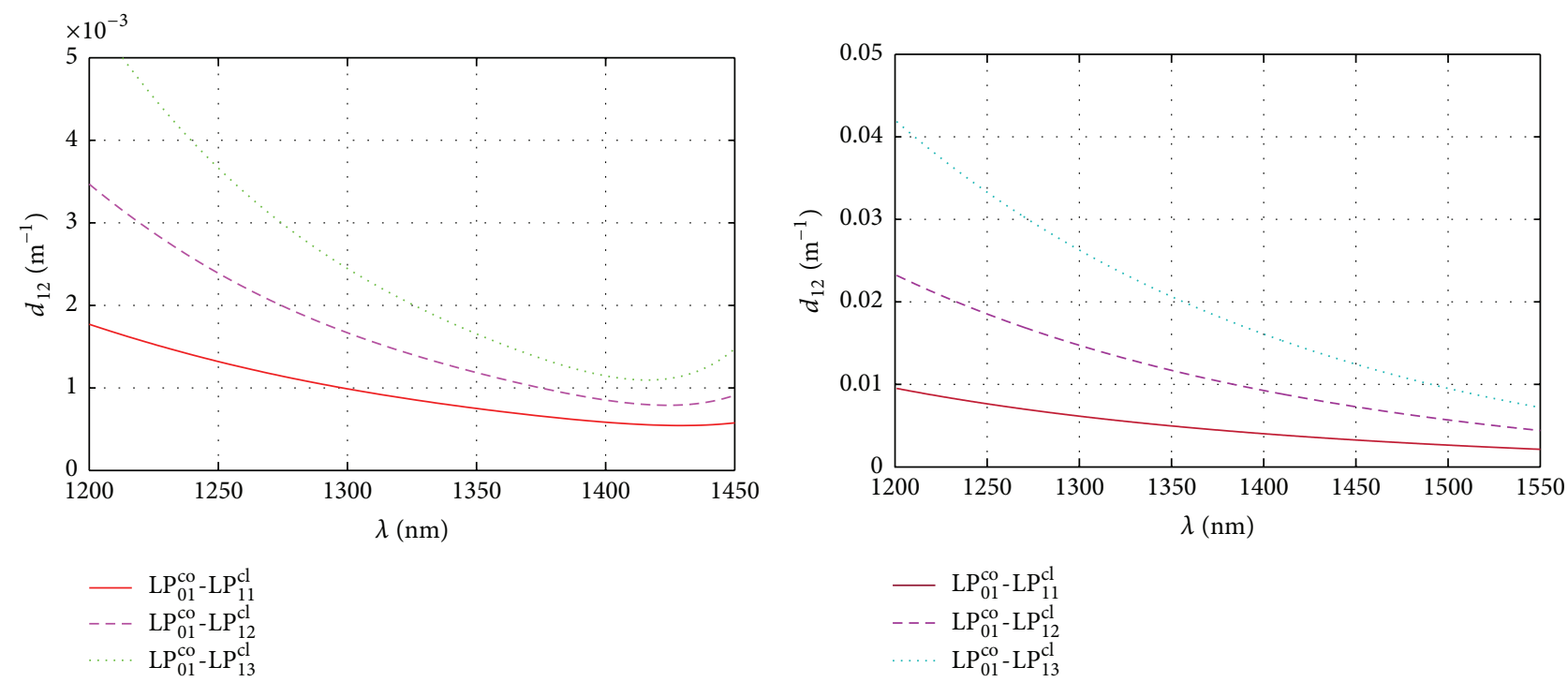

(a) $d_{\text {co }}=2.5 \mu \mathrm{m}$

(b) $d_{\mathrm{co}}=3.5 \mu \mathrm{m}$

FIGURE 8: Overlap field nonlinear coefficient $d_{12}$ as a function of wavelength in a tapered standard fiber. Two core diameters are considered, (a) $d_{\text {co }}=2.5 \mu \mathrm{m}$ and (b) $d_{\text {co }}=3.5 \mu \mathrm{m}$.

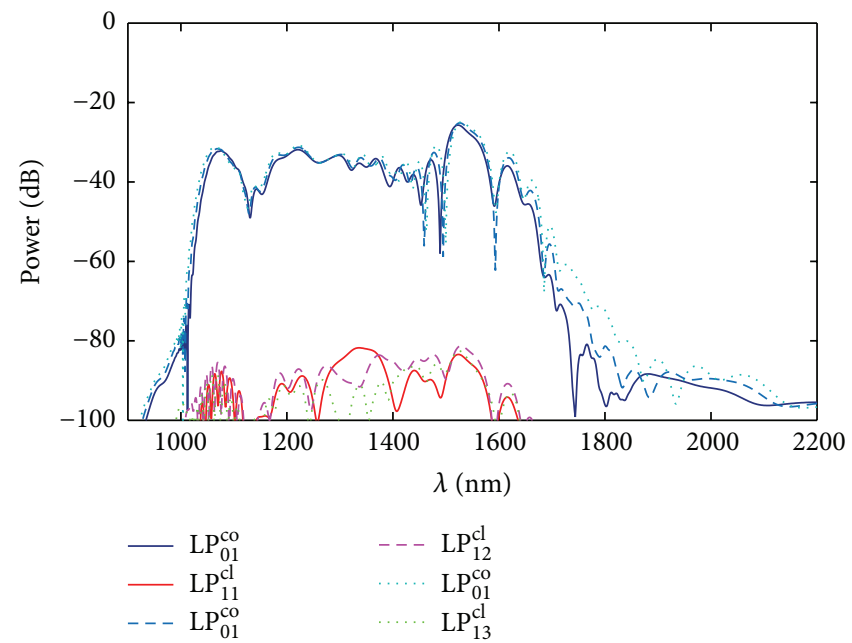

FIGURE 9: Nonlinear spectral response for $d_{\text {co }}=2.5 \mu \mathrm{m}$, with pump power of $P_{0}=30 \mathrm{~kW}$, initial width of $T_{0}=50 \mathrm{fs}$, and pump wavelength of $\lambda_{0}=1323 \mathrm{~nm}$.

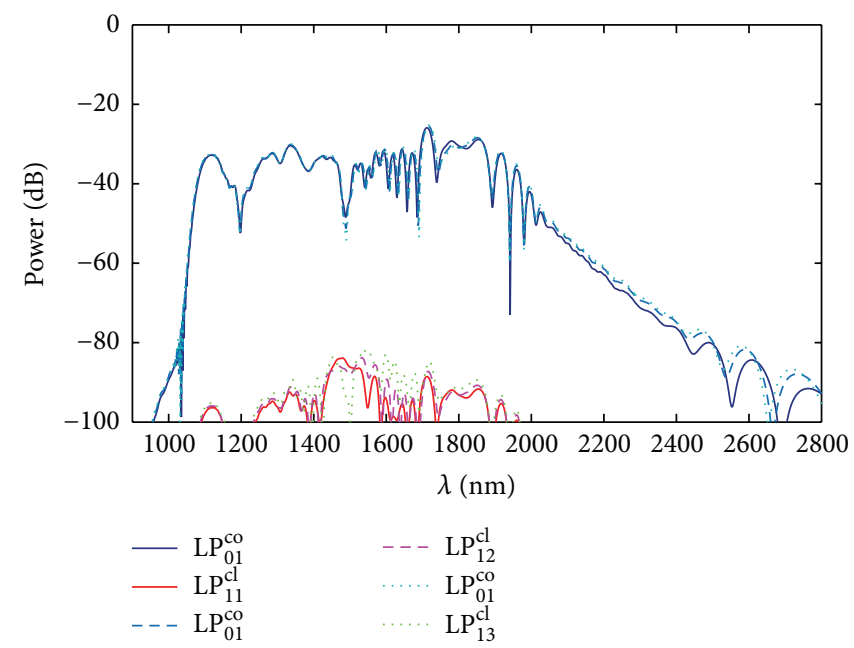

FIGURE 10: Nonlinear spectral response for $d_{\text {co }}=3.5 \mu \mathrm{m}$, with pump power of $P_{0}=3 \mathrm{~kW}$, initial width of $T_{0}=50 \mathrm{fs}$, and pump wavelength of $\lambda_{0}=1472 \mathrm{~nm}$. 


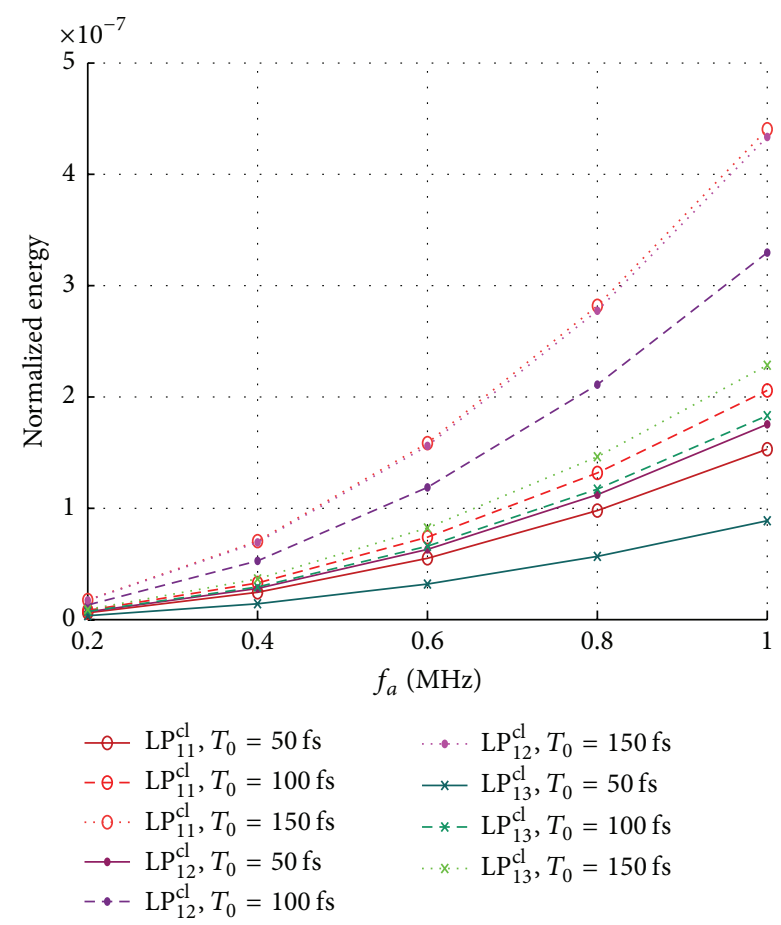

(a) $d_{\text {co }}=2.5 \mu \mathrm{m}$

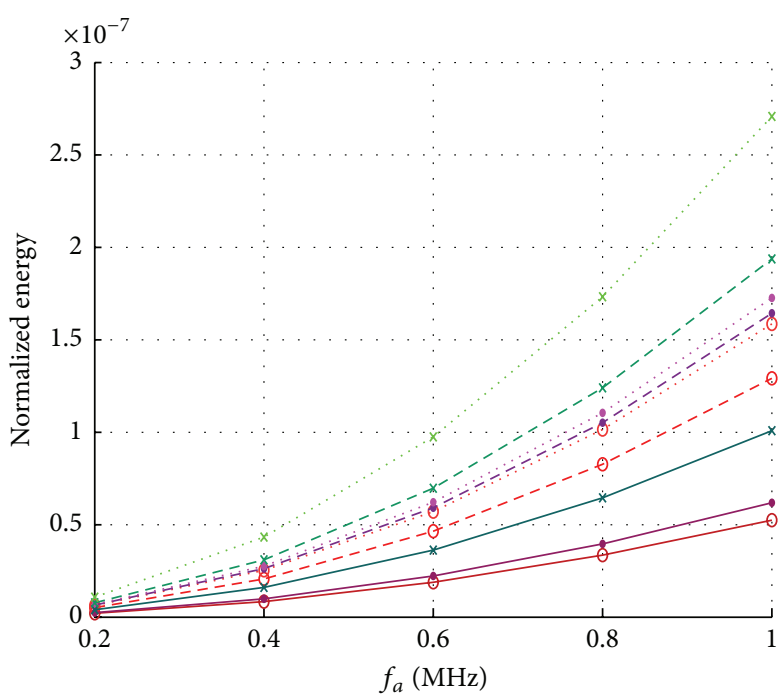

$$
\begin{array}{ll}
-0-\mathrm{LP}_{11}^{\mathrm{cl}}, T_{0}=50 \mathrm{fs} & \ldots . . \mathrm{LP}_{12}^{\mathrm{cl}}, T_{0}=150 \mathrm{fs} \\
-\ominus-\mathrm{LP}_{11}^{\mathrm{cl}}, T_{0}=100 \mathrm{fs} & -*-\mathrm{LP}_{13}^{\mathrm{cl}}, T_{0}=50 \mathrm{fs} \\
\cdots 0 . \mathrm{LP}_{11}^{\mathrm{cl}}, T_{0}=150 \mathrm{fs} & -*-\mathrm{LP}_{13}^{\mathrm{cl}}, T_{0}=100 \mathrm{fs} \\
\rightarrow-\mathrm{LP}_{12}^{\mathrm{cl}}, T_{0}=50 \mathrm{fs} & \ldots * \ldots \mathrm{LP}_{13}^{\mathrm{cl}}, T_{0}=150 \mathrm{fs} \\
-\bullet-\mathrm{LP}_{12}^{\mathrm{cl}}, T_{0}=100 \mathrm{fs} &
\end{array}
$$

(b) $d_{\text {co }}=3.5 \mu \mathrm{m}$

FIGURE 11: Normalized transferred energy from the core to the LP cladding modes as a function of acoustic frequency $f_{a}$ for different pulse width $T_{0}=50 \mathrm{fs}, 100 \mathrm{fs}$, and $150 \mathrm{fs}$. (a) For core diameter $2.5 \mu \mathrm{m}$ and (b) core diameter $3.5 \mu \mathrm{m}$.

TABLE 1: Dispersion coefficients $\mathrm{LP}_{01}^{\mathrm{co}}$ and LP cladding modes.

(a) $d_{\mathrm{co}}=2.5 \mu \mathrm{m}$ and $\lambda_{0}=1323 \mathrm{~nm}$

\begin{tabular}{lllll}
\hline Mode & $\beta_{2}\left[\mathrm{ps}^{2} \mathrm{~m}^{-1}\right]$ & $\beta_{3}\left[\mathrm{ps}^{3} \mathrm{~m}^{-1}\right]$ & $\beta_{4}\left[\mathrm{ps}^{4} \mathrm{~m}^{-1}\right]$ & $\beta_{5}\left[\mathrm{ps}^{5} \mathrm{~m}^{-1}\right]$ \\
\hline $\mathrm{LP}_{01}^{\mathrm{co}}$ & $-1.81 \times 10^{-4}$ & $1.05 \times 10^{-4}$ & $-1.66 \times 10^{-7}$ & $1.58 \times 10^{-10}$ \\
$\mathrm{LP}_{11}^{\mathrm{cl}}$ & $-4.67 \times 10^{-3}$ & $7.39 \times 10^{-5}$ & $-9.56 \times 10^{-8}$ & $9.69 \times 10^{-11}$ \\
$\mathrm{LP}_{12}^{\mathrm{cl}}$ & $-5.66 \times 10^{-3}$ & $7.57 \times 10^{-5}$ & $-9.85 \times 10^{-8}$ & $9.98 \times 10^{-11}$ \\
$\mathrm{LP}_{13}^{\mathrm{cl}}$ & $-6.94 \times 10^{-3}$ & $7.78 \times 10^{-5}$ & $-1.01 \times 10^{-7}$ & $1.02 \times 10^{-10}$ \\
\hline
\end{tabular}

(b) $d_{\text {co }}=3.5 \mu \mathrm{m}$ and $\lambda_{0}=1472 \mathrm{~nm}$

\begin{tabular}{lllll}
\hline Mode & $\beta_{2}\left[\mathrm{ps}^{2} \mathrm{~m}^{-1}\right]$ & $\beta_{3}\left[\mathrm{ps}^{3} \mathrm{~m}^{-1}\right]$ & $\beta_{4}\left[\mathrm{ps}^{4} \mathrm{~m}^{-1}\right]$ & $\beta_{5}\left[\mathrm{ps}^{5} \mathrm{~m}^{-1}\right]$ \\
\hline $\mathrm{LP}_{01}^{\mathrm{co}}$ & $-3.01 \times 10^{-4}$ & $1.01 \times 10^{-4}$ & $-1.89 \times 10^{-7}$ & $2.06 \times 10^{-10}$ \\
$\mathrm{LP}_{11}^{\mathrm{cl}}$ & $-1.86 \times 10^{-2}$ & $9.94 \times 10^{-5}$ & $-1.39 \times 10^{-7}$ & $1.33 \times 10^{-10}$ \\
$\mathrm{LP}_{12}^{\mathrm{cl}}$ & $-2.00 \times 10^{-2}$ & $1.02 \times 10^{-4}$ & $-1.43 \times 10^{-7}$ & $1.38 \times 10^{-10}$ \\
$\mathrm{LP}_{13}^{\mathrm{cl}}$ & $-2.18 \times 10^{-2}$ & $1.06 \times 10^{-4}$ & $-1.50 \times 10^{-7}$ & $1.44 \times 10^{-10}$ \\
\hline
\end{tabular}

even with less power compared to the $d_{\text {co }}=2.5 \mu \mathrm{m}$ case. This makes senses because $d_{12}$ is larger for $d_{\mathrm{co}}=3.5 \mu \mathrm{m}$ (see Figure 8).

As last step, we analyze how much energy can be transferred from the core to the LP cladding modes as a function of acoustic frequency $f_{a}$ for different pulse width $T_{0}=50 \mathrm{fs}$, $100 \mathrm{fs}$, and $150 \mathrm{fs}$; the results are plotted in Figure 11. The energy is normalized according to $E=P T / P_{0} T_{0}$, where $P T$ is computed as $\int_{-\infty}^{\infty} P(T) d T$. From Figure 11, the transferred energy behaves linearly increasing as $f_{a}$ and $T_{0}$ increase. This shows that although $C_{12}$ is proportional to $f_{a}^{2}$, the nonlinear effects linearize the energy and the slope can be controlled by $T_{0}$.

\section{Conclusions}

We have shown a complete procedure to study nonlinear pulse propagation in fiber optics when light is coupled from core mode to LP cladding modes as a consequence of acoustic wave. Based on local bending model, we introduce the concept of total variation of the refractive index as a sum of linear and nonlinear parts (Eqs. (13) and (14)). The procedure is applied to a tapered standard single mode fiber. We show that more energy can be nonlinearly transferred to the cladding if the tapered fiber diameter decreases. In addition, we have proven that the nonlinear effects compensate the coupling coefficients $\left(C_{12}\right)$ which is proportional to $f_{a}^{2}$; this makes the transferred energy to cladding be linearized with respect to acoustic frequency.

\section{Competing Interests}

The authors declare that they have no competing interests.

\section{Acknowledgments}

The authors acknowledge support from the Universidad Nacional de Colombia for this work (Grants nos. 17505, 25421, and 29900). 


\section{References}

[1] B. E. A. Saleh and M. C. Teich, Fundamentals of Photonics, John Wiley \& Sons, New York, NY, USA, 2nd edition, 1991.

[2] G. P. Agrawal, Fiber-Optic Communication Systems, John Wiley \& Sons, New York, NY, USA, 4th edition, 2002.

[3] Rp-photonics.com, "Encyclopedia of laser physics and technology-optical fiber communications," 2015, https://www.rpphotonics.com/optical_fiber_communications.html.

[4] S. M. Jensen, “The nonlinear coherent coupler," IEEE Journal of Quantum Electronics, vol. 18, no. 10, pp. 1580-1583, 1982.

[5] H. G. Winful, J. H. Marburger, and E. Garmire, "Theory of bistability in nonlinear distributed feedback structures," Applied Physics Letters, vol. 35, no. 5, pp. 379-381, 1979.

[6] J. N. Kutz, B. J. Eggleton, J. B. Stark, and R. E. Slusher, "Nonlinear pulse propagation in long-period fiber gratings: theory and experiment," IEEE Journal of Selected Topics in Quantum Electronics, vol. 3, no. 5, pp. 1232-1245, 1997.

[7] H. E. Engan, B. Y. Kim, J. N. Blake, and H. J. Shaw, "Propagation and optical interaction of guided acoustic waves in two-mode optical fibers," Journal of Lightwave Technology, vol. 6, no. 3, pp. 428-436, 1988.

[8] R. Acuna Herrera, C. Tai Law, and A. Mafi, "Calculation of the acousto-optic coupling coefficients in optical fibers," Optics Communications, vol. 305, pp. 217-220, 2013.

[9] H. Kogelnik, “Theory of optical waveguides," in Guided-Wave Optoelectronics, T. Tamir, Ed., vol. 26 of Springer Series in Electronics and Photonics, pp. 7-88, Springer, Berlin, Germany, 1988.

[10] T. Erdogan, "Cladding-mode resonances in short- and longperiod fiber grating filters," Journal of the Optical Society of America A: Optics and Image Science, and Vision, vol. 14, no. 8, pp. 1760-1773, 1997.

[11] O. V. Ivanov, S. A. Nikitov, and Y. V. Gulyaev, "Cladding modes of optical fibers: properties and applications," Physics-Uspekhi, vol. 49, no. 2, pp. 167-191, 2006.

[12] K. Okamoto, Fudamentals of Optical Waveguides, Elsevier, San Diego, Calif, USA, 2nd edition, 2006.

[13] J. Thomas, N. Jovanovic, R. G. Becker et al., "Cladding mode coupling in highly localized fiber Bragg gratings: modal properties and transmission spectra," Optics Express, vol. 19, no. 1, pp. 325-341, 2011.

[14] K. Karapetyan, Single optical microfibre-based modal interferometer [M.S. thesis], Universität Bonn, Bonn, Germany, 2012.

[15] V. E. Perlin and H. G. Winful, "Nonlinear pulse switching using long-period fiber gratings," Journal of Lightwave Technology, vol. 18, no. 3, pp. 329-333, 2000.

[16] G. P. Agrawal, Nonlinear Fiber Optics, chapter 2, Elsevier, San Diego, Calif, USA, 5th edition, 1989.

[17] P. Z. Dashti, Q. Li, and H. P. Lee, "Measurement of acoustic wavelength in optical fiber via acousto-optic interaction," Applied Physics Letters, vol. 81, no. 23, pp. 4338-4340, 2002.

[18] W. P. Risk, G. S. Kino, and B. T. Khuri-Yakub, "Tunable optical filter in fiber-optic form," Optics Letters, vol. 11, no. 9, pp. 578$580,1986$.

[19] D. Ostling and H. E. Engan, "Narrow-band acousto-optic tunable filtering in a two-mode fiber," Optics Letters, vol. 20, no. 11, pp. 1247-1249, 1995.

[20] H. S. Kim, S. H. Yun, I. K. Kwang, and B. Y. Kim, "All-fiber acousto-optic tunable notch filter with electronically controllable spectral profile," Optics Letters, vol. 22, no. 19, pp. 14761478, 1997. 


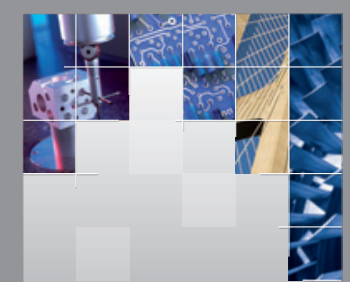

\section{Enfincering}
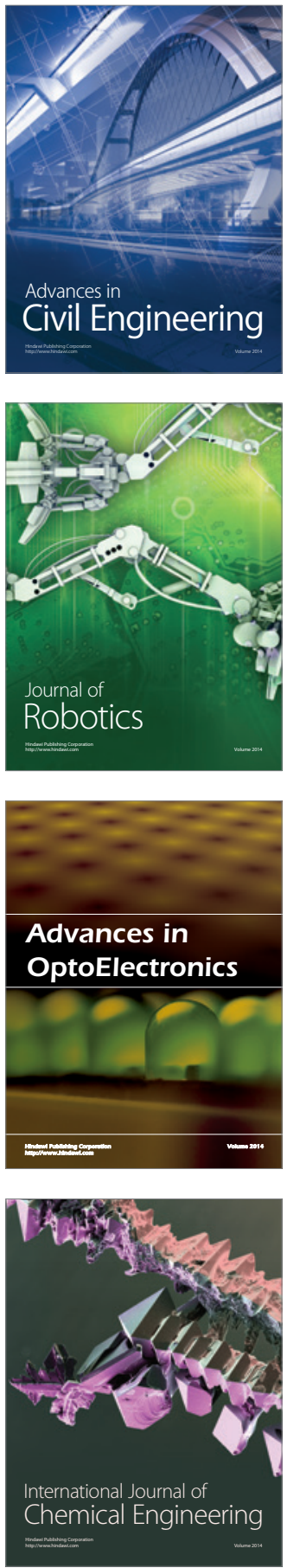

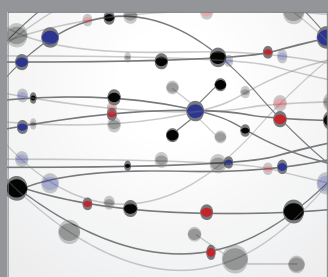

The Scientific World Journal

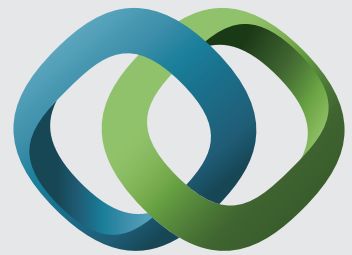

\section{Hindawi}

Submit your manuscripts at

http://www.hindawi.com
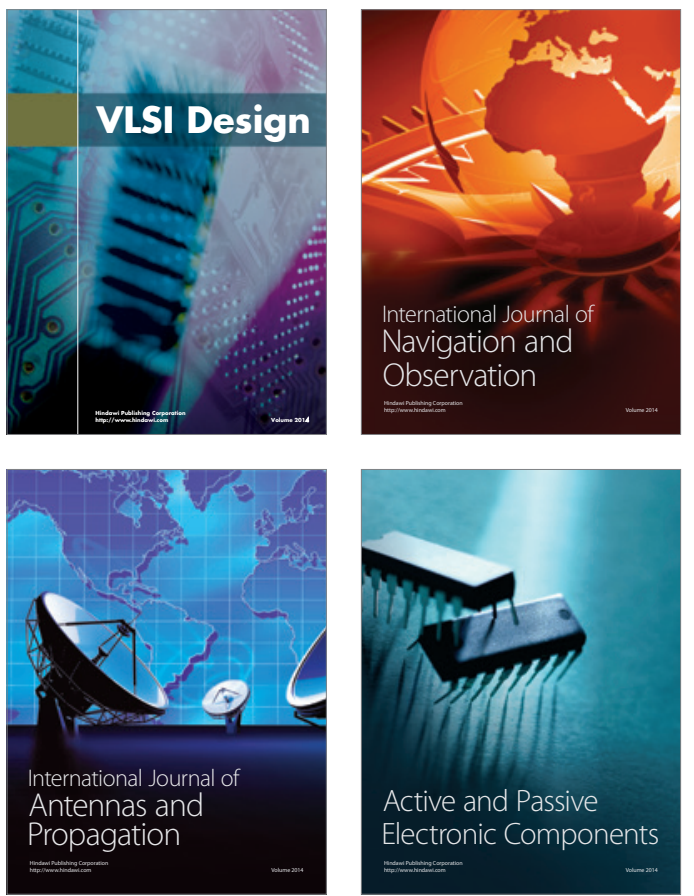
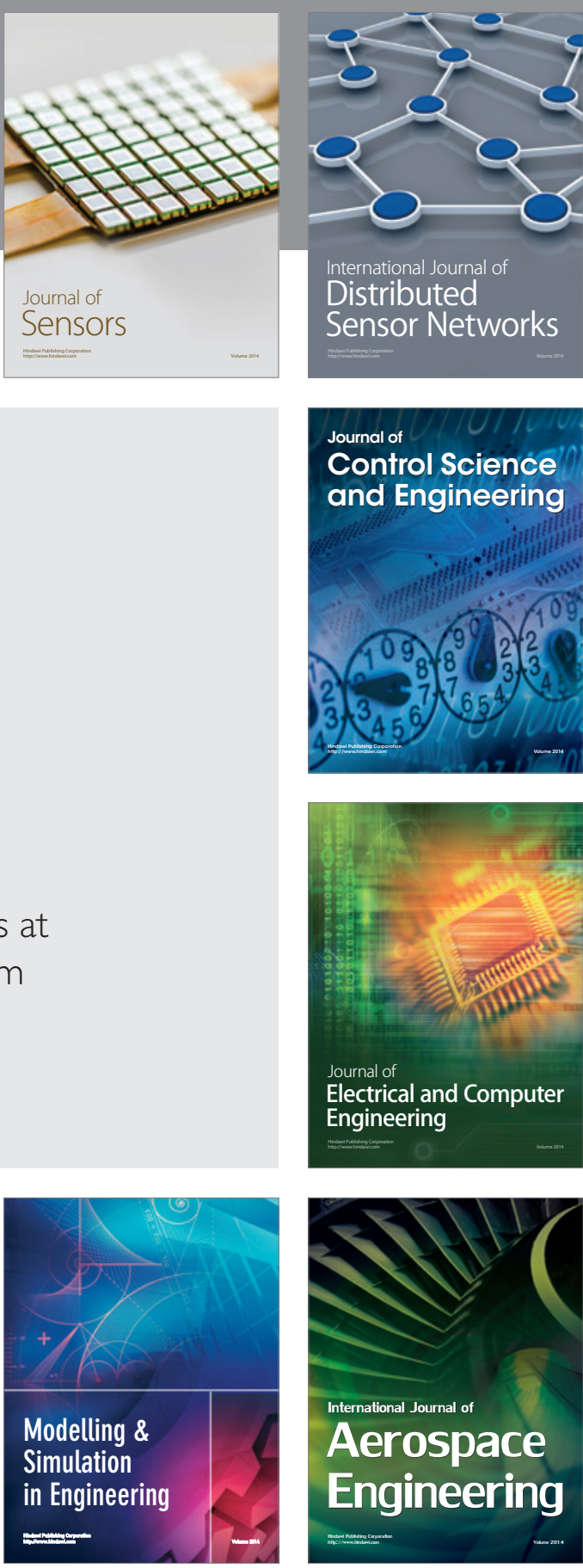

International Journal of

Distributed

Sensor Networks

Journal of

Control Science

and Engineering
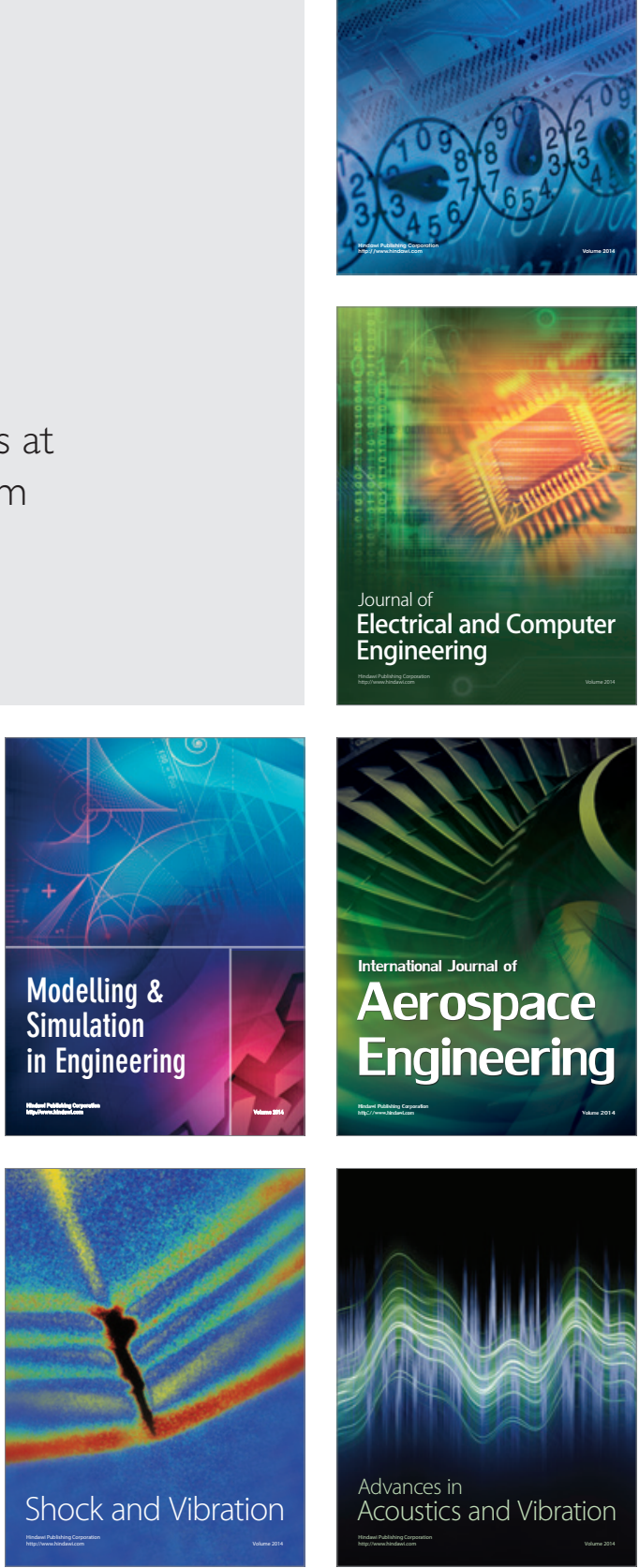